\title{
BIOSSEGURANÇA E RISCO OCUPACIONAL NA ATENÇÃO PRIMÁRIA: REVISÃO INTEGRATIVA DA LITERATURA
}

\author{
Luís Carlos de PAULA e SILVA ${ }^{1}$ \\ Carmen Maria Casquel Monti JULIANI ${ }^{2}$
}

\begin{abstract}
${ }^{1}$ Enfermeiro, Mestre em enfermagem, Diretor do Serviço de Enfermagem do Hospital das Clínicas, Docente da Faculdade de Medicina de Marília, Doutorando do curso de pós-graduação em Saúde Coletiva da Faculdade de Medicina da UNESP de Botucatu e Diretor do Departamento Regional de Saúde de Marília. Endereço para correspondência: Rua Tupinambás, 219, Jardim Aeroporto, Marília, SP, CEP: 17514-100, Telefone: (14) 97545113, email: luis@famema.br

${ }^{2}$ Enfermeira, Doutora em enfermagem, Docente dos cursos de Graduação e Pós-Graduação em Enfermagem e Docente do curso de pós-graduação em Saúde Coletiva da Faculdade de Medicina de Botucatu - UNESP. E-mail: cjuliani@fmb.unesp.br
\end{abstract}

Recebido em: 15/05/2014 - Aprovado em: 30/06/2014 - Disponibilizado em: 30/07/2014

\section{RESUMO}

Objetivou-se identificar, na produção científica mundial, os aspectos relacionados à biossegurança na atenção primária à saúde e as recomendações para um trabalho seguro. Utilizou-se a prática baseada em evidência, de Archie Cochrane, para a revisão da literatura, nas bases de dados da biblioteca Lilacs (Literatura Latino americana e do Caribe em Ciências da Saúde), PubMed, CINAHL e Scopus, sendo selecionados 32 artigos. Os profissionais que mais pesquisam sobre o assunto e os que mais têm participado das pesquisas são os enfermeiros, seguidos pelos médicos e os técnicos/auxiliares de enfermagem. Os riscos ocupacionais mais relatados fazem referência ao não uso de proteção. Houve aumento na produção científica sobre o assunto nos últimos anos, porém, na atenção primária, o tema é pouco discutido e pesquisado. Constatou-se, ainda, a não adesão às precauções padrão e a falta de treinamento, o que contribui para aumentar a exposição desses profissionais aos agentes biológicos.

Descritores: Riscos ocupacionais; Exposição a agentes biológicos; Atenção primária à saúde; Precauções universais; Saúde da família.

\section{BIOSAFETY AND OCCUPATIONAL RISK IN PRIMARY CARE: A LITERATURE REVIEW}

\begin{abstract}
This study aimed to identify the global scientific production, aspects related to biosafety in primary health care and recommendations for safe work. We used the evidence-based practice of Archie Cochrane to the literature review, the databases of the library Lilacs (Latin American and Caribbean Literature on Health Sciences), PubMed, CINAHL, and Scopus, being selected 32 articles. The professionals that most research on the subject and those who participated in the research are more nurses, followed by physicians and technicians/nursing assistants. Occupational hazards that are reported more make reference to not using protection. There was an increase in the scientific literature on the subject in recent years, but the primary theme is little discussed and researched. It was further observed, non-adherence to standard precautions and lack of training which contributes to increase exposure to biological agents such professionals. Keywords: Occupational risks; Exposure to biological agents; Primary health care; Universal precautions; Family health.
\end{abstract}




\section{Introdução}

No contexto da necessidade acentuada de ampliar o acesso dos usuários aos serviços de saúde, emerge uma nova realidade, traduzida pelo aumento na demanda à atenção primária, o que tem gerado desorganização do processo de trabalho e isso tem levado os profissionais que atuam nesse segmento à sobrecarga no cotidiano de suas atividades. Esse aumento na demanda surge da própria proposta do Sistema Único de Saúde (SUS), que busca, em suas políticas, ampliar o acesso das pessoas aos serviços de saúde. Além disso, com a proposta de se atingir maior resolubilidade, as ações tornaram-se mais complexas nos últimos anos.

Nessa nova forma de organização, em que a hierarquização se transforma em rede de atenção à saúde, de acordo com as diretrizes do SUS, a atenção primária tem um importante papel na implantação e viabilização das políticas de saúde no Brasil. Acontece que os profissionais que atuam neste segmento estão ampliando suas ações e muitos procedimentos invasivos vêm sendo desenvolvidos, o que tem acarretado exposição aos riscos biológicos, além de muitos desses procedimentos serem realizados em locais inadequados, que não oferecem condições ergonômicas ${ }^{(1-2)}$.

Assim, os profissionais da saúde estão expostos a acidentes com material biológico contaminado no desenvolvimento de suas atividades laborais. Alguns estudos mostram que os acidentes, com exposição do trabalhador a sangue e outros fluidos orgânicos, representam as ocorrências mais frequentes. Essas exposições são consideradas extremamente perigosas pela possibilidade de transmitir mais de 20 diferentes tipos de patógenos $^{(3-4-5-6-7-8-9)}$.

Diante disso, o risco ocupacional, após exposição a qualquer material biológico, depende do tipo de acidente, o tamanho da lesão, da presença e do volume de sangue, além das condições clínicas do paciente fonte e a instituição da profilaxia correta em tempo hábil $^{(5)}$.

No Brasil, os dados referentes aos acidentes com material biológico se assemelham aos de outros países. Outro dado nacional preocupante é que, de 36 a $45 \%$ dos profissionais que sofreram acidentes, procuraram assistência e fizeram a notificação desses acidentes, abandonaram o $\operatorname{tratamento}^{(10-11)}$.

Os riscos ocupacionais, que costumam acometer os trabalhadores das instituições de saúde são oriundos de fatores físicos, químicos, psicossocias, ergonômicos e biológicos $^{(12)}$. Esses últimos, os riscos biológicos, são provocados por agentes, tais como bactérias, fungos, bacilos, parasitas, protozoários e vírus ${ }^{(9)}$. 
Esses agentes são os responsáveis por provocar danos à saúde dos profissionais e isso se deve a característica das atividades executadas na área da saúde por exposição à material orgânico, ocorrendo a contaminação por via cutânea, respiratória ou digestiva ${ }^{(12-13)}$.

Com o intuito de entender essa realidade e tendo como base a contextualização do problema, este estudo tem a seguinte questão norteadora: Qual o conhecimento científico produzido acerca da biossegurança na atenção básica e quais as recomendações a serem observadas pelos trabalhadores para um trabalho seguro?

Portanto, este estudo tem sua relevância pautada na necessidade de explorar essa realidade em razão das poucas publicações existentes que apontem o cenário atual, principalmente nesse segmento específico, que é a atenção primária e, também, conhecer as estratégias para a promoção da saúde aos trabalhadores no que se refere aos riscos biológicos e à prevenção de danos à saúde. Além disso, esta pesquisa poderá subsidiar o desenvolvimento de práticas seguras para os profissionais que atuam na atenção à saúde.

Os estudos que abordam os acidentes biológicos estão, em sua maioria, relacionados aos ambientes hospitalares, na atenção secundária e terciária, sendo poucos os que tratam desses acidentes na atenção primária $^{(14-15-16-17)}$.

Em unidades de atenção primária à saúde, não se dispõe de dados mostrando diferenças e, portanto, uma grande lacuna de informações relacionadas ao tema. Dessa forma, é importante observar e analisar as atividades desenvolvidas nas diversas categorias, em unidades básicas de saúde integradas ao programa de saúde da família (PSF), considerando-se, de uma forma geral, o risco diferencial baseado no tipo e na frequência de procedimentos invasivos bem como a qualificação técnica desse profissional em relação à atividade sob sua responsabilidade. Acrescentam-se a esses fatores questões trabalhistas de responsabilidade profissional e as modalidades de vínculo empregatício no PSF, levado os profissionais a assumirem atividades em outras unidades de saúde de maior complexidade, com cargas de trabalho ampliadas e maior exposição aos agentes infecciosos e parasitários.

\section{Objetivo}

Em vista dessas razões, este estudo objetiva identificar a produção científica mundial relacionada à biossegurança na atenção primária à saúde e as recomendações para um trabalho seguro. 


\section{Método}

Trata-se de uma revisão integrativa da literatura mundial, tomando, como base, o referencial metodológico da prática baseada em evidência, de Archie Cochrane, que faz uma abordagem focada no cuidado clínico e no ensino fundamentado no conhecimento e na qualidade da evidência ${ }^{(18)}$.

A estratégia metodológica da revisão integrativa da literatura sintetiza estudos já concluídos para prover maior compreensão sobre um fenômeno específico ou um problema de saúde ${ }^{(19)}$. Esse método tem o potencial de construir conhecimento sobre as ciências da saúde e segue os mesmos critérios de uma pesquisa primária, ou seja, à clareza da metodologia, o rigor científico e a probabilidade de replicação ${ }^{(19)}$.

Com a definição do tema e da questão norteadora, arrolaram-se os artigos produzidos na literatura mundial. $\mathrm{O}$ levantamento foi realizado nos meses de outubro, novembro e dezembro de 2013, em periódicos da língua portuguesa, inglesa e espanhola, por meio da Biblioteca Virtual em Saúde (BVS) e Biblioteca Cochrane, disponibilizadas pelo Portal Capes. As bases de dados acessadas foram Lilacs (Literatura Latino americana e do Caribe em Ciências da Saúde), PubMed, CINAHL e Scopus.

Para a realização das buscas não houve delimitação de tempo das publicações, pois se trata de assunto escasso na literatura e, portanto, foram consideradas aquelas produzidas e que apresentavam relevância com o tema. Optou-se, inicialmente, pela utilização dos descritores padronizados para as ciências da saúde (www.decs.bvs.br) nos idiomas português, inglês e espanhol. Os descritores utilizados foram os seguintes: exposição a agentes biológicos; precauções universais; atenção primária à saúde; riscos ocupacionais e profissionais da saúde. Tais termos foram pesquisados nas bases de dados, isoladamente, para que fosse possível avaliar o poder de representatividade do descritor no assunto.

Na seleção das publicações e o critério de inclusão, foram considerados os artigos originais sobre a biossegurança e o risco biológico ocupacional relacionado aos profissionais que atuam na atenção primária da rede de atenção à saúde e o uso dos equipamentos de proteção individual (EPI) no desenvolvimento das atividades laborais.

Foram feitas as associações entre os descritores e, inicialmente, analisados os títulos das publicações. Daquelas que apresentavam relação com o tema, leu-se o resumo e, após leitura, se o artigo estivesse dentro dos critérios de inclusão, seria lido na íntegra para identificar se poderia ser utilizado no desenvolvimento do estudo. 
Inicialmente, então, fez-se a leitura dos resumos para identificar sua pertinência ao objeto estudado e, posteriormente, fez-se a busca dos artigos na íntegra, os quais foram lidos e analisados seguindo um roteiro elaborado pelos autores, contendo informações acerca de sua origem; ano de publicação; categoria profissional dos autores; local onde a pesquisa foi realizada; população estudada e, por fim, riscos ocupacionais identificados no trabalho nas unidades de Saúde Pública. Posteriormente, fez-se a interpretação das evidências oriundas dos artigos e sugestões para futuras pesquisas.

Para a análise criteriosa dos artigos, foi utilizada a classificação hierárquica das evidências, da Agency for Healthcare Research and Quality (AHRQ) dos EUA, a qual se baseia em seis níveis, a saber: nível 1metanálise de múltiplos estudos controlados; nível 2- estudo individual com desenho experimental; nível 3- estudo com desenho quase-experimental, séries temporais ou casocontrole; nível 4- pesquisa descritiva correlacional e qualitativa ou estudos de caso; nível 5- evidências provenientes de relatos de caso ou de experiência; nível 6- opinião de autoridades respeitáveis, baseada na competência clínica ou na opinião de comitês $^{(20)}$.

Entendemos que este estudo se encontra mais próximo ao nível 4 , conforme a classificação hierárquica das evidencias da
Agency for Healthcare Research and Quality

(AHRQ) dos EUA.

\section{Resultados e Discussão}

As informações encontradas nos 32 artigos selecionados para esta pesquisa subsidiaram a discussão e a conclusão e estão contidas no quadro abaixo de forma resumida.

Nesse quadro foram abordados os dados referentes ao título dos artigos, o periódico em que foram publicados, os autores responsáveis por sua elaboração, o ano de publicação e o país onde foi publicado. Foram, ainda, apresentados os delineamentos dos estudos, os profissionais que fizeram parte da pesquisa, os objetivos, os principais resultados e o nível de evidência. 


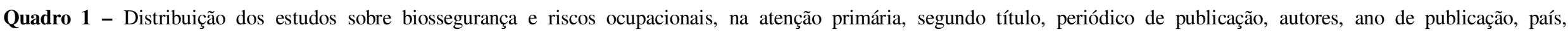
delineamento, profissionais pesquisados, objetivo do estudo, principais resultados e nível de evidência ( $\mathrm{N}=32)$.

\begin{tabular}{|c|c|c|c|c|c|}
\hline Título/Periódico & Autores/Ano/Pais & Delineamento/Profissionais & Objetivos & $\begin{array}{l}\text { Principais } \\
\text { Resultados }\end{array}$ & $\begin{array}{l}\text { Nível de } \\
\text { Evidência }\end{array}$ \\
\hline $\begin{array}{l}\text { O risco de infecção entre os } \\
\text { profissionais de saúde primários } \\
\text { na região de desenvolvimento } \\
\text { ocidental, Nepal: o conhecimento } \\
\text { e cumprimento. } \\
\text { Nepal: knowledge } \\
\text { compliance. } \\
\text { J Infect Dev Ctries }\end{array}$ & $\begin{array}{l}\text { Timilshina N, Ansari MA, Dayal V } \\
2011 \\
\text { Nepal - India }\end{array}$ & $\begin{array}{l}\text { Estudo quantitativo e prospectivo } \\
\text { com } 100 \text { trabalhadores da saúde. }\end{array}$ & $\begin{array}{l}\text { Avaliar a conformidade dos } \\
\text { procedimentos de controle de } \\
\text { infecção nas unidades de } \\
\text { cuidados de saúde primários. }\end{array}$ & $\begin{array}{l}\text { O estudo mostrou o } \\
\text { não uso regular de } \\
\text { luvas de proteção } \\
\text { durante o manuseio de } \\
\text { pacientes. }\end{array}$ & III \\
\hline $\begin{array}{l}\text { Percepção do risco no trabalho em } \\
\text { saúde da família: estudo com } \\
\text { trabalhadores no sul do Brasil } \\
\text { Rev. latinoam. Enferm }\end{array}$ & $\begin{array}{l}\text { Cezar-Vaz MR, Soares JF, } \\
\text { Figueiredo PP, Azambuja EP, } \\
\text { Sant'anna CF, Costa VZ. } \\
2009 \\
\text { Brasil }\end{array}$ & 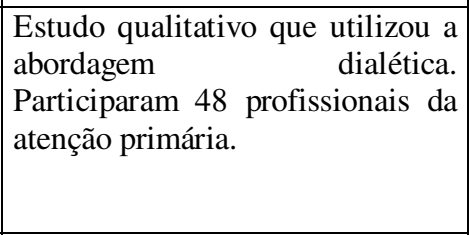 & $\begin{array}{l}\text { Identificar a percepção } \text { dos } \\
\text { trabalhadores da Estratégia em } \\
\text { Saúde da Família sobre os } \\
\text { riscos aos quais estão } \\
\text { expostos. }\end{array}$ & $\begin{array}{l}\text { O estudo mostrou que } \\
\text { os riscos percebidos } \\
\text { foram róridentes } \\
\text { biológicos, desgaste } \\
\text { emocional e falta de } \\
\text { resolução no trabalho. }\end{array}$ & IV \\
\hline $\begin{array}{l}\text { Segurança na aplicação de injeção } \\
\text { na atenção primária no sudoeste } \\
\text { da Arábia Saudita. } \\
\text { East Mediterr Health J. }\end{array}$ & $\begin{array}{l}\text { Mahfouz AA, Abdelmoneim I, } \\
\text { Khan MY, Daffalla AA, Diab MM, } \\
\text { Shaban H, Al Amri HS. } \\
2009 \\
\text { Arabia Saudita }\end{array}$ & $\begin{array}{l}\text { Estudo quantitativo prospectivo. } \\
\text { Participantes: } 156 \text { profissionais } \\
\text { da atenção primária. }\end{array}$ & 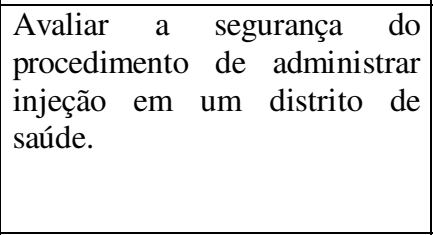 & $\begin{array}{l}\text { O estudo identificou } \\
\text { que recapar a agulha } \\
\text { após o uso e dobrá-la } \\
\text { antes do descarte são } \\
\text { fatores de risco para as } \\
\text { lesões. }\end{array}$ & III \\
\hline $\begin{array}{l}\text { Exposição a sangue e fluídos } \\
\text { corporais em cuidados primários } \\
\text { de saúde brasileira. } \\
\text { Occup Med (Lond) }\end{array}$ & $\begin{array}{l}\text { Garcia LP, Facchini LA } \\
2009 \\
\text { Brasil }\end{array}$ & $\begin{array}{l}\text { Estudo quantitativo e transversal. } \\
\text { Participantes: } 1077 \text { trabalhadores } \\
\text { da atenção primária de saúde. }\end{array}$ & $\begin{array}{|lcrr|}\text { Investigar } & \text { a } & \text { exposição a } \\
\text { sangue e } & \text { fluídos } & \text { corporais } \\
(B B F) \text { e } & \text { seus } & \text { fatores } \\
\text { associados } & \text { entre } & \text { os } \\
\text { profissionais } & \text { de } & \text { saúde } \\
\text { primários. } & & \\
\end{array}$ & $\begin{array}{l}\text { O estudo mostrou que } \\
\text { o auxiliar de } \\
\text { enfermagem, o } \\
\text { assistente de dentista e } \\
\text { o dentista estão } \\
\text { expostos a acidentes } \\
\text { de trabalho pelas } \\
\text { condições inadequadas } \\
\text { de trabalho e o } \\
\text { excesso de atividades. }\end{array}$ & III \\
\hline $\begin{array}{l}\text { O impacto das intervenções } \\
\text { educativas no conhecimento da }\end{array}$ & $\begin{array}{l}\text { Krishnan P, Dick F, Murphy E. } \\
2007\end{array}$ & \begin{tabular}{|l} 
Ensaio clínico randomizado. \\
Participantes: 1.120 dentistas da
\end{tabular} & \begin{tabular}{|lrrr} 
Avaliar & o & impacto & de \\
intervenções & educativas & sobre
\end{tabular} & $\begin{array}{l}\text { O estudo mostrou que } \\
\text { o treinamento corpo a }\end{array}$ & I \\
\hline
\end{tabular}




\begin{tabular}{|c|c|c|c|c|c|}
\hline $\begin{array}{l}\text { exposição ocupacional a sangue e } \\
\text { fluídos corporais dos } \\
\text { trabalhadores de cuidados de } \\
\text { saúde primários. } \\
\text { Occup Med (Lond) }\end{array}$ & Escócia & atenção primária. & \begin{tabular}{|l} 
cuidados primários de saúde \\
no conhecimento e gestão da \\
exposição ocupacional dos \\
trabalhadores a sangue $\quad$ e \\
fluidos corporais.
\end{tabular} & \begin{tabular}{|lrr} 
corpo foi & a \\
intervenção & mais \\
eficaz & quando \\
comparado com & as \\
outras medidas. &
\end{tabular} & \\
\hline $\begin{array}{l}\text { Risco biológico em cuidados de } \\
\text { enfermagem prestados nas } \\
\text { unidades de saúde da família. } \\
\text { Rev. Latino-Am. Enfermagem }\end{array}$ & $\begin{array}{l}\text { Cardoso ACM, Figueiredo RM. } \\
2010 \\
\text { Brasil }\end{array}$ & $\begin{array}{l}\text { Estudo quantitativo. Participaram } \\
\text { do estudo profissionais de } 12 \\
\text { unidades de PSF. }\end{array}$ & \begin{tabular}{llr|} 
Identificar as & situações onde \\
há riscos & de exposição \\
biológica para & profissionais de \\
enfermagem & duranter a \\
assistência de enfermagem em \\
unidades de PSF.
\end{tabular} & \begin{tabular}{ll}
\multicolumn{3}{l}{ O estudo mostrou que } \\
os profissionais da \\
atenção primária estão \\
sujeitos a riscos \\
semelhantes aos dos \\
trabalhadores & \\
hospitalares, & pela \\
exposição a & sangue e \\
frequência & na \\
manipulação & de \\
agulhas. &
\end{tabular} & III \\
\hline $\begin{array}{l}\text { Questões de saúde ocupacional } \\
\text { dos profissionais de saúde bucal } \\
\text { no Estado de Edo, na Nigéria. } \\
\text { Int J Dent }\end{array}$ & $\begin{array}{l}\text { Osazuwa-Peters N, } \\
\text { Azodo CC, } \\
\text { Obuekwe ON. } \\
\text { Nigéria } \\
2012\end{array}$ & $\begin{array}{l}\text { Estudo quantitativo transversal. } \\
\text { Os participantes foram os } \\
\text { profissionais de saúde bucal. }\end{array}$ & $\begin{array}{l}\text { Avaliar a percepção e as } \\
\text { prevalências de problemas de } \\
\text { saúde ocupacionais entre os } \\
\text { trabalhadores de saúde bucal } \\
\text { no Estado de Edo, na Nigéria. }\end{array}$ & $\begin{array}{l}\text { O estudo mostrou que } \\
\text { os riscos biológicos } \\
\text { representam o maior } \\
\text { problema de saúde } \\
\text { ocupacional para os } \\
\text { dentistas. }\end{array}$ & III \\
\hline $\begin{array}{l}\text { A percepção dos dentistas sobre } \\
\text { riscos ocupacionais e medidas } \\
\text { preventivas em Jerusalém } \\
\text { Oriental. } \\
\text { East Mediterr Health J. }\end{array}$ & $\begin{array}{l}\text { Al-Khatib IA, } \\
\text { Ishtayeh M, } \\
\text { Barghouty H, } \\
\text { Akkawi B. } \\
2006 \\
\text { Israel }\end{array}$ & $\begin{array}{l}\text { Estudo quantitativo. Os } \\
\text { participantes foram } 40 \text { dentistas. }\end{array}$ & $\begin{array}{l}\text { Identificar a percepção dos } \\
\text { riscos ocupacionais e medidas } \\
\text { preventivas em } \text { em Jerusalém } \\
\text { Oriental dos dentistas e e } \\
\text { determinar se são empregadas } \\
\text { técnicas preventivas. }\end{array}$ & $\begin{array}{l}\text { O estudo demonstrou } \\
\text { que a maioria dos } \\
\text { entrevistados estava } \\
\text { ciente dos riscos } \\
\text { biológicos, sendo a } \\
\text { hepatite B e o vírus da } \\
\text { imunodeficiência } \\
\text { humana os que mais } \\
\text { preocupam. }\end{array}$ & III \\
\hline $\begin{array}{l}\text { Riscos ocupacionais do } \\
\text { enfermeiro atuante na estratégia } \\
\text { saúde da família } \\
\text { Rev. enferm. UERJ }\end{array}$ & $\begin{array}{l}\text { Bessa MEP, Almeida MI, Araújo } \\
\text { MFM, Silva MJ. } \\
2010 \\
\text { Brasil }\end{array}$ & Estudo de revisão bibliográfica. & $\begin{array}{l}\text { Discutir os riscos ocupacionais } \\
\text { a que os enfermeiros atuantes } \\
\text { na ESF estão expostos, } \\
\text { segundo a literatura. }\end{array}$ & $\begin{array}{l}\text { O estudo demonstrou } \\
\text { que os enfermeiros } \\
\text { estão expostos a riscos } \\
\text { físicos, químicos e } \\
\text { biológicos (secreções } \\
\text { oral, vaginal e de }\end{array}$ & IV \\
\hline
\end{tabular}




\begin{tabular}{|c|c|c|c|c|c|}
\hline & & & & $\begin{array}{|lrr|}\text { feridas). } & \text { Percebe-se } \\
\text { que } & \text { os } & \text { riscos } \\
\text { biológicos } & \text { estão } & \text { entre } \\
\text { os } & \text { temas } & \text { mais } \\
\text { evidenciados } & \text { na } \\
\text { literatura. } & \\
\end{array}$ & \\
\hline $\begin{array}{l}\text { Riscos ocupacionais dos } \\
\text { enfermeiros atuantes na atenção à } \\
\text { saúde da família. } \\
\text { Rev. enferm. UERJ }\end{array}$ & $\begin{array}{l}\text { Nunes MBG, Robazzi MLCC, Terra } \\
\text { FS, Mauro M YC, Zeitoune RCG, } \\
\text { Secco IAO. } \\
2010 \\
\text { Brasil }\end{array}$ & $\begin{array}{|llr|}\text { Estudo quantitativo e qualitativo. } \\
\text { Participaram do estudo } 20 \\
\text { enfermeiros atuantes em } 9 \\
\text { unidades de saúde da família. }\end{array}$ & $\begin{array}{l}\text { Descrever os riscos } \\
\text { ocupacionais dos enfermeiros } \\
\text { que atuam na rede básica de } \\
\text { saúde, estratégia da saúde da } \\
\text { família. }\end{array}$ & $\begin{array}{|lr|}\text { O estudo demonstrou } \\
\text { que o risco biológico } \\
\text { esta entre os riscos } \\
\text { ocupacionais } & \\
\text { identificados } & \text { pelos } \\
\text { enfermeiros. } & \end{array}$ & IV \\
\hline $\begin{array}{l}\text { Representações de profissionais } \\
\text { da atenção primária sobre risco } \\
\text { ocupacional de infecção pelo HIV. } \\
\text { Rev. Latino-Am. Enfermagem }\end{array}$ & $\begin{array}{l}\text { Souza MCMR, Freitas MIF. } \\
2010 \\
\text { Brasil }\end{array}$ & $\begin{array}{l}\text { Estudo qualitativo fundamentado } \\
\text { na Teoria das Representações } \\
\text { Sociais. Participaram médicos e } \\
\text { enfermeiros das USF e das } \\
\text { equipes de apoio das unidades. }\end{array}$ & $\begin{array}{l}\text { Analisar as representações de } \\
\text { profissionais de saúde que } \\
\text { atuam na atenção primária } \\
\text { sobre o risco de infecção pelo } \\
\text { HIV, ao qual estão expostos no } \\
\text { cotidiano do trabalho. }\end{array}$ & \begin{tabular}{|lr}
\multicolumn{3}{l}{ O estudo mostrou que, } \\
embora & os \\
profissionais de & saúde \\
conheçam o risco de \\
infecção em & seu \\
cotidiano de trabalho, \\
representando-o romo \\
muito $r$ baixo \\
atenção básica, na não \\
aderem as precauções.
\end{tabular} & IV \\
\hline $\begin{array}{l}\text { Risco de infecção tuberculosa em } \\
\text { agentes comunitários de saúde. } \\
\text { Rev Saúde Pública }\end{array}$ & $\begin{array}{l}\text { Moreira TR, Maciel EZELN. } \\
2010 \\
\text { Brasil }\end{array}$ & $\begin{array}{|lrr|}\text { Estudo de } & \text { coorte } & \text { prospectivo. } \\
\text { Participaram } & 61 & \text { agentes } \\
\text { comunitários. } & & \\
\end{array}$ & 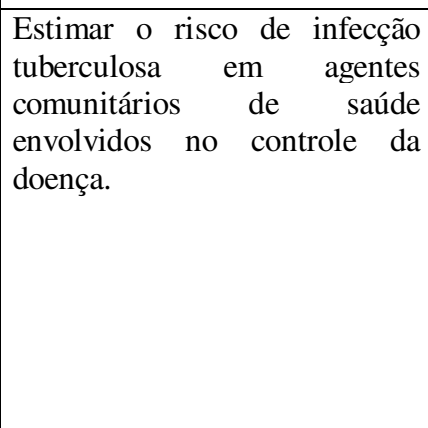 & \begin{tabular}{|lr} 
O estudo mostrou que \\
os agentes \\
acompanharam \\
pacientes \\
tuberculose durante \\
sua rotina de trabalho \\
apresentaram um risco \\
maior de infecção do \\
que aqueles que não \\
acompanharam esses \\
pacientes.
\end{tabular} & I \\
\hline $\begin{array}{l}\text { Situação vacinal e sorológica para } \\
\text { hepatite B em profissionais da } \\
\text { estratégia saúde da família. }\end{array}$ & $\begin{array}{l}\text { Oliveira VC, Guimarães } \text { EAA, } \\
\text { Souza DAS, Ricardo RA. } \\
2011 \\
\text { Brasil }\end{array}$ & $\begin{array}{|lrr|}\text { Estudo } & & \text { quantitativo } \\
\text { epidemiológico. Participaram } 175 \\
\text { profissionais da saúde de } 15 \\
\text { USF. }\end{array}$ & $\begin{array}{l}\text { Verificar a situação vacinal } \\
\text { contra hepatite B e a re- } \\
\text { alização de sorologia para anti- } \\
\text { HBS em profissionais da }\end{array}$ & 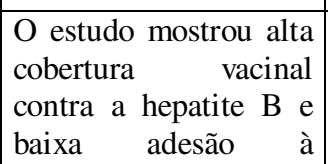 & III \\
\hline
\end{tabular}

269

Revista da Universidade Vale do Rio Verde, Três Corações, v. 12, n. 1, p. 262-281, jan./jul. 2014 


\begin{tabular}{|c|c|c|c|c|c|}
\hline Rev Rene & & USF. & $\begin{array}{l}\text { Estratégia Saúde da Família } \\
\text { (ESF), em Divinópolis, MG. }\end{array}$ & sorologia. & \\
\hline $\begin{array}{l}\text { Condutas pós-acidentes } \\
\text { perfurocortantes: percepção e } \\
\text { conhecimento de enfermeiros da } \\
\text { atenção básica de Fortaleza. } \\
\text { Rev Rene }\end{array}$ & $\begin{array}{l}\text { Feijão AR, Martins LHFA, Marques } \\
\text { MB. } \\
2011 \\
\text { Brasil }\end{array}$ & $\begin{array}{l}\text { Estudo quantitativo. Participaram } \\
36 \text { enfermeiros que atuam nas } \\
\text { Unidades Básicas de Saúde. }\end{array}$ & $\begin{array}{l}\text { Investigar o conhecimento dos } \\
\text { enfermeiros } \\
\text { a sobre as condutas } \\
\text { acidenter comadas diante de um } \\
\text { perfurocortante. }\end{array}$ & $\begin{array}{l}\text { O estudo mostrou que } \\
\text { os enfermeiros se } \\
\text { consideraram } \\
\text { conhecedores das } \\
\text { condutas e sentem-se } \\
\text { preparados para agir } \\
\text { diante de um acidente } \\
\text { de trabalho. }\end{array}$ & III \\
\hline 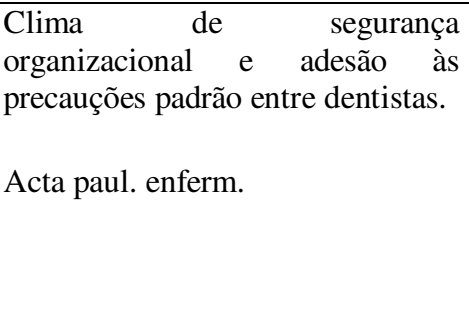 & $\begin{array}{l}\text { Ribeiro PHV, Brevidelli MM, } \\
\text { Tipple AFV, Ribeiro RP, Elucir G. } \\
2013 \\
\text { Brasil }\end{array}$ & $\begin{array}{l}\text { Estudo quantitativo. Participaram } \\
224 \text { dentistas que atuavam em } \\
\text { unidades de Atenção Básica de } \\
\text { Saúde. }\end{array}$ & $\begin{array}{l}\text { Verificar a percepção } \text { do } \\
\text { dentista a respeito do clima de } \\
\text { segurança no trabalho em } \\
\text { relação à adesão às precauções } \\
\text { padrão. }\end{array}$ & $\begin{array}{l}\text { O estudo mostrou que } \\
\text { o clima de segurança } \\
\text { insatisfatório e a falta } \\
\text { de treinamento em } \\
\text { saúde ocupacional } \\
\text { favorecem a não } \\
\text { adesão as precauções } \\
\text { padrão. }\end{array}$ & III \\
\hline $\begin{array}{l}\text { A vacinação contra hepatite B é } \\
\text { realidade entre trabalhadores da } \\
\text { Atenção Primária à Saúde? } \\
\text { Rev. Latino-Am. Enfermagem }\end{array}$ & $\begin{array}{l}\text { Costa FM, Martins AMEBL, Neto } \\
\text { PES, Veloso DNP, Magalhães VS, } \\
\text { Ferreira RC. } \\
2013 \\
\text { Brasil }\end{array}$ & $\begin{array}{l}\text { Estudo quantitativo transversal. } \\
\text { Participaram } 797 \text { profissionais da } \\
\text { atenção primária. }\end{array}$ & $\begin{array}{l}\text { Investigar a prevalência e os } \\
\text { fatores associados à vacinação } \\
\text { contra hepatite B, entre } \\
\text { trabalhadores da APS, } \\
\text { analisando, simultaneamente, } \\
\text { fatores considerados } \\
\text { importantes na determinação } \\
\text { da vacinação contra hepatite } \\
\text { B, que não foram previamente } \\
\text { estudados. }\end{array}$ & $\begin{array}{l}\text { O estudo demonstrou } \\
\text { que a prevalência e os } \\
\text { fatores associados à } \\
\text { vacinação foram } \\
\text { maiores em pessoas } \\
\text { com mais anos de } \\
\text { escolaridade e aqueles } \\
\text { que relataram contato } \\
\text { com instrumentos } \\
\text { cortantes. }\end{array}$ & III \\
\hline $\begin{array}{l}\text { Análise da susceptibilidade às } \\
\text { doenças imunopreveníveis em } \\
\text { profissionais de saúde a partir do } \\
\text { status vacinal. } \\
\text { Rev Rene }\end{array}$ & $\begin{array}{l}\text { Pinto ACS, Almeida MI, Pinheiro } \\
\text { PNC } \\
2011 \\
\text { Brasil }\end{array}$ & $\begin{array}{l}\text { Estudo quantitativo. Participaram } \\
47 \text { profissionais que atuavam nas } \\
\text { unidades básicas de saúde. }\end{array}$ & $\begin{array}{l}\text { Descrever o status vacinal de } \\
\text { todos os profissionais de nível } \\
\text { superior atuantes nas unidades } \\
\text { da ESF no Município de } \\
\text { Morada Nova/ Ceará. }\end{array}$ & $\begin{array}{l}\text { O estudo mostrou que } \\
\text { os profissionais estão } \\
\text { vulneráveis aos riscos } \\
\text { ocupacionais em razão } \\
\text { de não estarem com o } \\
\text { esquema vacinal } \\
\text { completo. }\end{array}$ & III \\
\hline
\end{tabular}




\begin{tabular}{|c|c|c|c|c|c|}
\hline $\begin{array}{l}\text { Riscos ocupacionais: percepção } \\
\text { de profissionais de enfermagem } \\
\text { da estratégia saúde da família em } \\
\text { João Pessoa - PB } \\
\text { R bras ci Saúde }\end{array}$ & $\begin{array}{l}\text { Rodrigues LMC, Silva CCS, Silva } \\
\text { VKBA, Martiniano CS, Silva ACO, } \\
\text { Martins MO. } \\
2012 \\
\text { Brasil }\end{array}$ & $\begin{array}{l}\text { Estudo qualitativo. Participaram } \\
12 \text { profissionais de enfermagem } \\
\text { de quatro unidades de saúde da } \\
\text { família de João Pessoa/PB. }\end{array}$ & $\begin{array}{l}\text { Investigar a compreensão de } \\
\text { trabalhadores de enfermagem } \\
\text { de Unidades de Saúde da } \\
\text { Família quanto aos riscos } \\
\text { ocupacionais a que se } \\
\text { encontram expostos } \\
\text { identificar suas sugestões para } \\
\text { minimizar tal exposição. }\end{array}$ & \begin{tabular}{l}
\multicolumn{3}{l}{ O estudo mostrou que } \\
as condições de \\
trabalho, a falta de \\
educação em saúde e o \\
número inadequado de \\
profissionais estão \\
associados r à \\
exposição aos riscos \\
ocupacionais.
\end{tabular} & IV \\
\hline $\begin{array}{l}\text { Risco e vulnerabilidade } \\
\text { práticas dos profissionais } \\
\text { saúde } \\
\text { Rev Gaúcha Enferm }\end{array}$ & $\begin{array}{l}\text { Santos JLG, Vieira M, Assuiti LFC, } \\
\text { Gomes D, Meirelles BHS, Santos } \\
\text { SMA. } \\
2012 \\
\text { Brasil }\end{array}$ & $\begin{array}{l}\text { Estudo de revisão integrativa da } \\
\text { literatura, constituindo uma } \\
\text { amostra de } 21 \text { publicações na } \\
\text { atenção primária à saúde. }\end{array}$ & $\begin{array}{l}\text { Analisar a produção científica } \\
\text { da área de saúde e } \\
\text { enfermagem acerca dos fatores } \\
\text { de risco e da vulnerabilidade } \\
\text { nas práticas dos profissionais } \\
\text { de saúde, por meio de uma } \\
\text { revisão integrativa. }\end{array}$ & $\begin{array}{l}\text { O estudo mostrou que } \\
\text { na atenção primária à } \\
\text { saúde, os riscos e as } \\
\text { vulnerabilidades } \\
\text { relacionam-se à } \\
\text { deficiência de recursos } \\
\text { para o trabalho, à } \\
\text { violência física e ao } \\
\text { desgaste emocional. }\end{array}$ & IV \\
\hline \begin{tabular}{lccc} 
Precauções & \multicolumn{2}{c}{ básicas } & e \\
gerenciamento & de & resíduos & na \\
coleta para & o & exame & de \\
papanicolau. & & & \\
Rev. Rene & & &
\end{tabular} & $\begin{array}{l}\text { Oliveira NC, Moura } \\
\text { ERF. } \\
2009 \\
\text { Brasil }\end{array}$ & $\begin{array}{l}\text { Estudo qualitativo. Participaram } \\
21 \text { unidades de saúde da família } \\
\text { de Maranguape-CE, sendo } \\
\text { observados } 63 \text { procedimentos } \\
\text { realizados por enfermeiros. }\end{array}$ & 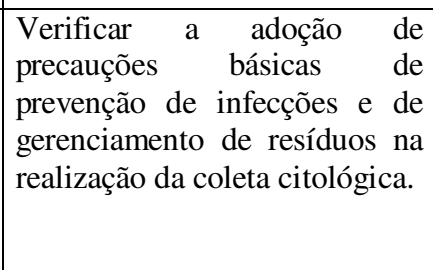 & $\begin{array}{l}\text { O estudo mostrou que } \\
\text { a lavagem das mãos } \\
\text { não é um hábito das } \\
\text { enfermeiras, embora } \\
\text { tenham utilizado luvas } \\
\text { em todos os } \\
\text { procedimentos. }\end{array}$ & IV \\
\hline $\begin{array}{l}\text { Riscos ocupacionais } \\
\text { trabalhadores de }\end{array}$ & $\begin{array}{l}\text { Chiodi MB, Marziale MHP. } \\
2006 \\
\text { Brasil }\end{array}$ & $\begin{array}{l}\text { Estudo bibliográfico. A amostra } \\
\text { foi constituída por } 12 \text { publicações } \\
\text { que abordam os riscos } \\
\text { ocupacionais em Saúde Pública. }\end{array}$ & $\begin{array}{l}\text { Buscar evidências científicas } \\
\text { na literatura nacional sobre os } \\
\text { riscos ocupacionais a que estão } \\
\text { expostos os trabalhadores que } \\
\text { atuam na Saúde Pública. }\end{array}$ & $\begin{array}{l}\text { O estudo mostrou que } \\
\text { os riscos psicossociais } \\
\text { foram evidenciados } \\
\text { em todas as pesquisas, } \\
\text { seguidos pelo risco } \\
\text { biológico. }\end{array}$ & IV \\
\hline $\begin{array}{l}\text { Risco ocupacional enfrentado } \\
\text { pelos enfermeiros que atuam na } \\
\text { atenção primária à saúde. } \\
\text { J Nurs UFPE on line }\end{array}$ & 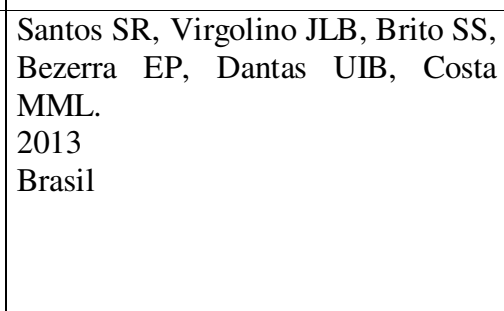 & $\begin{array}{l}\text { Estudo quantitativo. Participaram } \\
53 \text { enfermeiros que atuam nas } \\
\text { Unidades de Saúde da Família, } \\
\text { do Distrito Sanitário III, em João } \\
\text { Pessoa/PB/Brasil. }\end{array}$ & $\begin{array}{l}\text { Analisar a concepção dos } \\
\text { enfermeiros que atuam na } \\
\text { Atenção Primária à Saúde } \\
\text { acerca das medidas de } \\
\text { biossegurança em seu processo } \\
\text { de trabalho. }\end{array}$ & 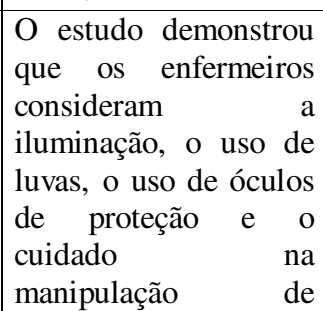 & III \\
\hline
\end{tabular}




\begin{tabular}{|c|c|c|c|c|c|}
\hline & & & & \begin{tabular}{|lr} 
perfurocortante & como \\
medidas & de \\
biossegurança. & \\
\end{tabular} & \\
\hline $\begin{array}{l}\text { Fatores de riscos ocupacionais } \\
\text { para sangue e fluídos corporais: } \\
\text { exposição entre assessores de } \\
\text { cuidados em casa. } \\
\text { Home Health Care Services } \\
\text { Quarterly }\end{array}$ & $\begin{array}{l}\text { Amuwo S, Shakirudeen A, Sokas } \\
\text { RK, McPhaul K, Lipscomb J. } \\
2011 \\
\text { USA }\end{array}$ & $\begin{array}{l}\text { Estudo quantitativo. Participaram } \\
\text { assessores de home care. }\end{array}$ & $\begin{array}{l}\text { Avaliar a exposição } \\
\text { ocupacional auto-relatada a } \\
\text { sangue e fluidos corporais para } \\
\text { determinar se os fatores que } \\
\text { colocam os trabalhadores em } \\
\text { situação de risco podem ser } \\
\text { identificados. }\end{array}$ & $\begin{array}{l}\text { O estudo mostrou que } \\
\text { os profissionais estão } \\
\text { expostos a sangue e } \\
\text { fluidos corporais, por } \\
\text { meio de objetos } \\
\text { cortantes ou contato } \\
\text { com mucosas. }\end{array}$ & III \\
\hline $\begin{array}{l}\text { Morte devido a infecção } \\
\text { transmitida pelo sangue e suas } \\
\text { sequelas entre os trabalhadores da } \\
\text { saúde. } \\
\text { Am J Ind Med }\end{array}$ & $\begin{array}{l}\text { Luckhaupt SE, Calvert GM. } \\
2008 \\
\text { USA }\end{array}$ & $\begin{array}{l}\text { Estudo caso-controle pareado que } \\
\text { utilizou o banco de dados da } \\
\text { Agência Nacional de Vigilância } \\
\text { Ocupacional e } \\
\text { (NOMS). }\end{array}$ & $\begin{array}{l}\text { Avaliar as chances de morrer } \\
\text { por doenças transmissíveis } \\
\text { entre trabalhadores da área da } \\
\text { saúde. }\end{array}$ & $\begin{array}{l}\text { O estudo mostrou que } \\
\text { a enfermagem foi a } \\
\text { ocupação com os mais } \\
\text { altos riscos de morte, } \\
\text { entre os homens para } \\
\text { HIV e HBV, mas as } \\
\text { enfermeiras } \\
\text { apresentavam } \\
\text { diminuição do risco de } \\
\text { morte por HIV. }\end{array}$ & III \\
\hline $\begin{array}{l}\text { Os riscos da exposição de } \\
\text { enfermeiros não hospitalares aos } \\
\text { agents patogênicos. } \\
\text { Ind Health }\end{array}$ & $\begin{array}{l}\text { Gershon RR, Qureshi KA, } \\
\text { Pogorzelska M, Rosen J, Gebbie } \\
\text { KM, Brandt Rauf PW, Sherman } \\
\text { MF. } \\
2007 \\
\text { USA }\end{array}$ & \begin{tabular}{|l}
\multicolumn{3}{|c|}{ Estudo qualitativo e quantitativo. } \\
Participaram enfermeiros \\
atenção básica \\
RNs.
\end{tabular} & $\begin{array}{l}\text { Avaliar o risco de exposição a } \\
\text { sangue e fluidos corporais } \\
\text { entre os enfermeiros não- } \\
\text { hospitalares, que trabalham no } \\
\text { Estado de Nova Iorque. }\end{array}$ & $\begin{array}{l}\text { O estudo mostrou que } \\
\text { os profissionais estão } \\
\text { expostos a acidentes } \\
\text { com agulhas. As } \\
\text { subnotificações são } \\
\text { comuns. O tratamento } \\
\text { pós-exposição não é } \\
\text { praticado na maioria } \\
\text { dos casos. }\end{array}$ & IV \\
\hline $\begin{array}{l}\text { Transmissão ocupacional de } \\
\text { doenças transmitidas pelo sangue } \\
\text { para os trabalhadores da saúde nos } \\
\text { países em desenvolvimento: } \\
\text { enfrentar os desafios. } \\
\text { Journal of Hospital Infection }\end{array}$ & $\begin{array}{l}\text { Lee R. } \\
2009 \\
\text { Reino Unido }\end{array}$ & $\begin{array}{l}\text { Estudo bibliográfico. A amostra } \\
\text { foi constituída por artigos do } \\
\text { Medline e Web of Science com } \\
\text { combinações de palavras-chave: } \\
\text { HIV, hepatite B, hepatite C, os } \\
\text { trabalhadores de saúde, a } \\
\text { exposição ocupacional, acidentes } \\
\text { com agulhas; profilaxia pós- }\end{array}$ & $\begin{array}{l}\text { Investigar os riscos de } \\
\text { transmissão de três patógenos: } \\
\text { vírus da imunodeficiência } \\
\text { humana, hepatite B e vírus da } \\
\text { hepatite C, e identificar os } \\
\text { fatores que influenciam o risco } \\
\text { em relação aos países em } \\
\text { desenvolvimento. }\end{array}$ & $\begin{array}{l}\text { O estudo mostrou que } \\
\text { as precauções padrão, } \\
\text { em que todos os } \\
\text { pacientes são } \\
\text { infectados e onde as } \\
\text { práticas de proteção } \\
\text { são adotadas tem-se } \\
\text { mostrado efetivas na }\end{array}$ & IV \\
\hline
\end{tabular}




\begin{tabular}{|c|c|c|c|c|c|}
\hline & & \begin{tabular}{llr|} 
exposição; & atitudes & dos \\
profissionais de saúde, a a & de \\
segurança da injeção; países em \\
desenvolvimento. Para as buscas \\
também foram utilizado o \\
gateway ELDIS e ra da \\
Organização Mundial da Saúde \\
(OMS) e os Centros de Controle \\
e Prevenção de Doenças (CDC) \\
site.
\end{tabular} & & 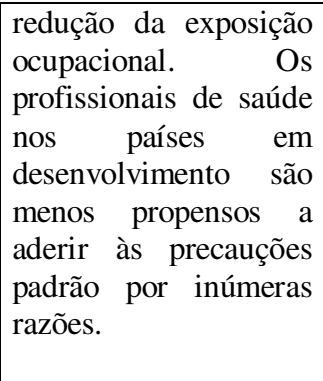 & \\
\hline $\begin{array}{l}\text { A exposição ocupacional a } \\
\text { acidentes com agulhas e cobertura } \\
\text { vacinal contra hepatite B entre } \\
\text { trabalhadores de saúde do Egito. } \\
\text { American Journal of Infection } \\
\text { Control }\end{array}$ & $\begin{array}{l}\text { Talaat M, Kandeel A, El-Shoubary } \\
\text { W, Bodenschatz C, Khairy I, Oun S, } \\
\text { MB BCh, Mahoney FJ. } \\
2003 \\
\text { Egito }\end{array}$ & $\begin{array}{|llr|}\text { Estudo quantitativo. } & \text { Participaram } \\
1.485 & \text { profissionais de saúde, } \\
\text { sendo coletados } & \text { dados sobre } & \text { a } \\
\text { história de } & \text { exposição } & \text { a } \\
\text { ferimentos com } & \text { agulhas, estado } \\
\text { vacinal e dados socioeconômicos. }\end{array}$ & $\begin{array}{l}\text { Identificar a frequência de } \\
\text { exposição a acidentes com } \\
\text { agulhas e cobertura vacinal } \\
\text { contra a hepatite } B \text { entre os } \\
\text { profissionais de saúde no } \\
\text { Egito. }\end{array}$ & $\begin{array}{lr}\text { O estudo mostrou que, } \\
\text { dos } & 1.485 \\
\text { profissionais de } & \text { saúde } \\
\text { entrevistados, } & 529 \\
(35,6 \%) & \text { foram } \\
\text { expostos a pelo menos } \\
\text { um ferimento por } \\
\text { picada de agulha } \\
\text { durante os últimos três } \\
\text { meses. A cobertura } \\
\text { vacinal foi maior entre } \\
\text { os profissionais de } \\
\text { nível superior, e } \\
\text { menor entre } \\
\text { funcionários os } \\
\text { limpeza. }\end{array}$ & IV \\
\hline $\begin{array}{l}\text { Lesões agudas e seus } \\
\text { determinantes entre os } \\
\text { profissionais de saúde em serviços } \\
\text { de cuidados de primeiro nível na } \\
\text { Província de Sindh, no Paquistão. } \\
\text { Tropical Medicine and } \\
\text { International Health }\end{array}$ & $\begin{array}{l}\text { Naveed Z, Janjua MI. Khan BM. } \\
2010 \\
\text { Paquistão }\end{array}$ & $\begin{array}{l}\text { Estudo quantitativo. Participaram } \\
233 \text { profissionais de saúde em } \\
\text { clínicas gerais, públicas e e } \\
\text { privadas, na atenção primária. }\end{array}$ & $\begin{array}{llrr}\text { Avaliar a } & \text { taxa } & \text { e } & \text { os } \\
\text { determinantes } & \text { das } & \text { lesões } \\
\text { agudas durante os seis } & \text { meses } \\
\text { anteriores, entre profissionais } \\
\text { de saúde nas unidades de } \\
\text { cuidados de primeiro nível em } \\
\text { dois distritos do Paquistão. }\end{array}$ & $\begin{array}{l}\text { O estudo mostrou que } \\
54 \% \text { dos } 233 \\
\text { trabalhadores tiveram, } \\
\text { pelo menos, uma lesão } \\
\text { durante os seis meses } \\
\text { anteriores. }\end{array}$ & III \\
\hline $\begin{array}{l}\text { Um estudo comparativo sobre a } \\
\text { identificação dos riscos } \\
\text { ocupacionais por trabalhadores de } \\
\text { enfermagem de duas Unidades } \\
\text { Básicas de Saúde do município de }\end{array}$ & $\begin{array}{l}\text { Silva RCG, Felli VEA. } \\
2002 \\
\text { Brasil }\end{array}$ & $\begin{array}{l}\text { Estudo quantitativo. Participaram } \\
33 \text { profissionais de enfermagem } \\
\text { de duas Unidades Básicas de } \\
\text { Saúde. }\end{array}$ & 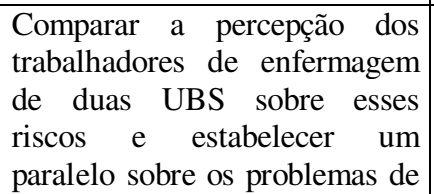 & \begin{tabular}{|lr|}
\multicolumn{3}{|l}{ O estudo mostrou que, } \\
com relação & à \\
percepção & da \\
exposição aos & riscos \\
ocupacionais, & os
\end{tabular} & III \\
\hline
\end{tabular}




\begin{tabular}{|c|c|c|c|c|c|}
\hline $\begin{array}{l}\text { São Paulo } \\
\text { Rev. esc. enferm. USP }\end{array}$ & & & $\begin{array}{l}\text { saúde relacionados com a sua } \\
\text { exposição. }\end{array}$ & $\begin{array}{l}\text { trabalhadores de } \\
\text { ambas UBS referiram, } \\
\text { com frequência maior, } \\
\text { a exposição aos riscos } \\
\text { biológicos. }\end{array}$ & \\
\hline $\begin{array}{l}\text { Segurança do trabalho, entre os } \\
\text { trabalhadores de saúde poloneses } \\
\text { em relação à exposição à } \\
\text { patógenos. } \\
\text { Med Pr. }\end{array}$ & $\begin{array}{l}\text { Rvbacki M, } \\
\text { Piekarska A, } \\
\text { Wiszniewska M, } \\
\text { Walusiak SJ. } \\
2013 \\
\text { Polônia }\end{array}$ & $\begin{array}{l}\text { Estudo quantitativo. Participaram } \\
242 \text { trabalhadores da área de } \\
\text { saúde polonesa. }\end{array}$ & $\begin{array}{l}\text { Avaliar a eficácia dos métodos } \\
\text { que são usados para promover } \\
\text { a segurança do trabalho nos } \\
\text { serviços de saúde, para avaliar } \\
\text { a frequêencia de exposição nos } \\
\text { últimos } 12 \text { meses anteriores ao } \\
\text { estudo e determinar uma taxa } \\
\text { para denunciá-los as } \\
\text { autoridades. }\end{array}$ & $\begin{array}{lr}\text { O estudo mostrou que } \\
\text { a exposição acidental } \\
\text { percutânea } \\
\text { ocupacional durante } \\
\text { os últimos } 12 \text { meses, } \\
\text { foi declarada pelos } \\
242 \text { trabalhadores } \\
(21 \% \text { de todo o } \\
\text { grupo). Apenas } & \text { em } \\
146 \text { casos } & \text { esses } \\
\text { incidentes } & \text { foram } \\
\text { relatados } & \text { às } \\
\text { autoridades. } & \\
\text { Incidentes } & \text { de } \\
\text { exposição } & \text { foram } \\
\text { associados } & \text { com } \\
\text { autopercepção de alto } \\
\text { risco de exposição. } \\
\end{array}$ & IIII \\
\hline $\begin{array}{l}\text { Accidentes biológicos con los } \\
\text { profesionales de salud en la } \\
\text { atención primaria de Guadalajara } \\
\text { (1994-2000) } \\
\text { Acidentes biológicos de trabalho } \\
\text { com profissionais de saúde da } \\
\text { atenção primária em Guadalajara } \\
\text { (1994-2000) } \\
\text { Rev. Salud Ambient }\end{array}$ & $\begin{array}{l}\text { López CJ, De la Rosa RG, Prieto } \\
\text { MAD, Mato SA, Fernandez SMMI. } \\
2011 \\
\text { Espanha }\end{array}$ & $\begin{array}{l}\text { Estudo quantitativo. Participaram } \\
\text { profissionais da atenção primária } \\
\text { de saúde em Guadalajara. }\end{array}$ & 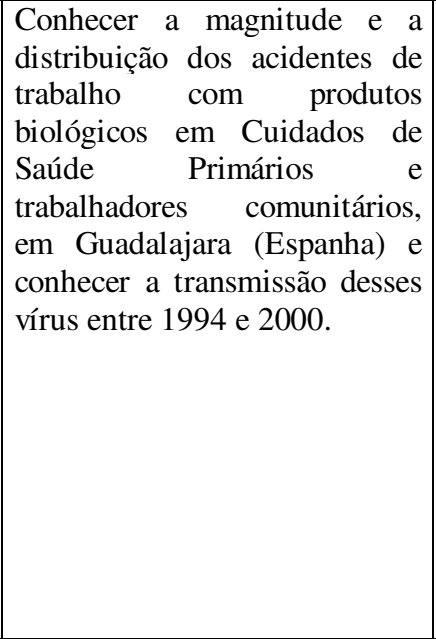 & 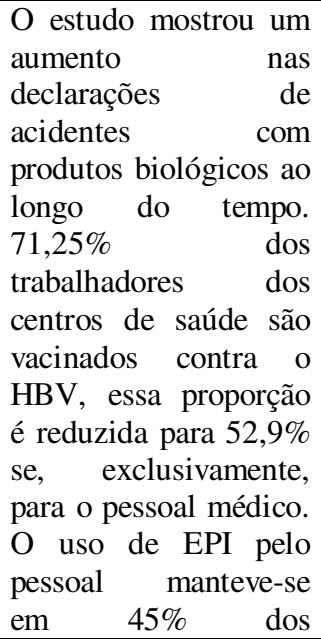 & IIII \\
\hline
\end{tabular}




\begin{tabular}{|c|c|c|c|c|c|}
\hline & & & & $\begin{array}{l}\text { trabalhadores no } \\
\text { momento do acidente. } \\
\text { Não se observou } \\
\text { nenhuma infecção em } \\
\text { profissionais de saúde } \\
\text { ou na população em } \\
\text { geral durante o estudo } \\
\text { no período de } \\
\text { monitoramento. }\end{array}$ & \\
\hline $\begin{array}{l}\text { O profissional de saúde na } \\
\begin{array}{l}\text { Atenção } \\
\text { metassíntese }\end{array} \\
\text { Sau. \& Transia: } \\
\text { uma }\end{array}$ & $\begin{array}{l}\text { Marilise K. } \\
2011 \\
\text { Brasil }\end{array}$ & $\begin{array}{l}\text { Estudo de revisão da bibliografia. } \\
\text { A amostra foi constituída por } \\
\text { artigos localizados nas bases de } \\
\text { dados LILACS, Pubmed, SciELO } \\
\text { e os descritores "primary health } \\
\text { care" e "qualitative research". }\end{array}$ & 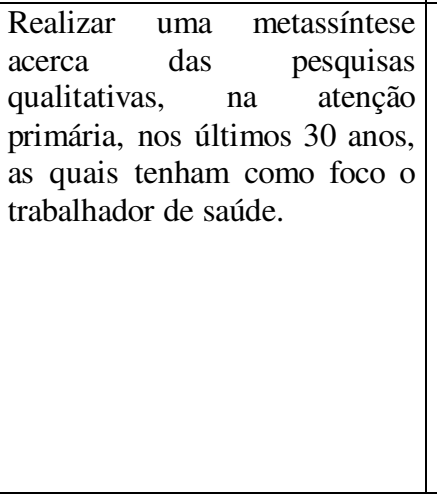 & $\begin{array}{l}\text { O estudo identificou } 6 \\
\text { artigos que abordaram } \\
\text { os fatores de risco aos } \\
\text { profissionais da saúde } \\
\text { no âmbito da Atenção } \\
\text { Primária (AP) com } \\
\text { relação a saúde do } \\
\text { trabalhador. } \\
\text { Constatou-se que a } \\
\text { literatura é escassa, } \\
\text { apesar do crescimento } \\
\text { de publicações com } \\
\text { abordagem qualitativa. }\end{array}$ & IV \\
\hline
\end{tabular}


Dos 32 artigos que compuseram este estudo (5/32) foram obtidos por meio das bases de dados Lilacs e corresponderam a $15,6 \%$; no PubMed (8/32), a 25\%; no CINAHL (10/32), a $31,2 \%$ e no Scopus $(9 / 32)$, a $28,1 \%$. As bases de dados que mais apresentaram artigos relacionados ao assunto foram o Scopus, com $28,1 \%$ e o CINAHL, com $31,2 \%$.

$\mathrm{Na}$ classificação hierárquica das evidências da Agency for Healthcare Research and Quality (AHRQ) dos EUA, os artigos selecionados que compõem o quadro anterior apresentaram os níveis de evidência I (2/32), II (0/32), III (18/32), IV (12/32), V (0/32) e VI (0/32), concentrando-se, em sua maioria nos níveis de evidencia III e IV.

Os profissionais que pesquisaram sobre o assunto foram 15,6\% dentistas (5/32); $50 \%$ enfermeiros (16/32); $3,1 \%$ fonoaudiólogo (1/32); 28,1\% médicos (9/32) e 3,1\% psicólogo (1/32). Os artigos mostram que os profissionais que mais desenvolveram pesquisas sobre a biossegurança na atenção primária foram os enfermeiros, com $50 \%$ e os médicos com $28,1 \%$.

Os profissionais que participaram dos estudos, considerando a frequência com que cada categoria apareceu em cada um dos artigos foram dentistas em 12 artigos, enfermeiros 22, médicos 11 , técnicos de enfermagem 8, auxiliares de enfermagem 12 , auxiliares de dentista 6 e agentes comunitários 2. Os estudos mostram que os profissionais mais pesquisados na atenção primária, no que se refere ao risco biológico, são os enfermeiros, os médicos e os auxiliares e técnicos de enfermagem. A literatura aponta que os profissionais mais expostos a acidentes biológicos, na área da saúde, são os profissionais da enfermagem, principalmente o nível técnico ${ }^{(21)}$. Outro estudo realizado em hospitais públicos mostra que os dentistas, médicos e técnicos de laboratório têm-se acidentado mais em contrapartida a farmacêuticos e enfermeiros ${ }^{(22)}$.

Quanto ao ano de publicação dos artigos, ocorreram de 6,3\% de 2002 a 2005 (2/32); 15,6\% de 2006 a 2008 (5/32); 53,1\% de 2009 a $2011(17 / 32)$ e $25 \%$ a partir de 2012 (8/32). Quanto aos locais de realização das pesquisas, aconteceram $65,6 \%$ nas unidades básicas de saúde (21/32) e 34,4\%, nas unidades de saúde da família. Nota-se que o período em que se obtiveram mais publicações foi de 2009 a 2011.

Quanto ao delineamento das pesquisas, $56,3 \%$ são estudos quantitativos $(18 / 32) ; 12,5 \%$, qualitativos $(4 / 32) ; 3,1 \%$, ensaio clinico randomizado (1/32); 15,6\%, revisão bibliográfica $(5 / 32), 6,3 \%$, estudos qualiquantitativos $(2 / 32) ; 3,1 \%$, estudos de coorte prospectivos (1/32) e 3,1\%, estudo caso controle (1/32). Pelos dados, a pesquisa quantitativa foi a mais utilizada pelos 
pesquisadores para abordar a biossegurança na atenção primária.

Os riscos ocupacionais biológicos identificados, considerando a frequência com que cada tipo de risco expõe o profissional durante suas atividades laborais, assim se evidenciaram: 6 acidentes provocados por agulhas; 13 em razão de não usarem luvas ou outros equipamentos de proteção durante os procedimentos; 3 por excesso de trabalho; 7 por falta de treinamento; 5 estão relacionados a não lavar as mãos; 8 pela falta de imunização dos profissionais; 5 por destinação inadequada dos perfuro cortantes e 3 por falta de material adequado para o trabalho. Nessa abordagem, fica evidente que a exposição aos riscos biológicos está diretamente ligada ao não uso dos equipamentos de proteção individual e à falta de treinamento.

A baixa adesão ao uso dos Equipamentos de Proteção Individual (EPI) sofre influência de aspectos comportamentais, tais como o desconforto durante o uso e a dificuldade para realizar determinados procedimentos $^{(23)}$.

Com relação à distribuição geográfica (locais onde as pesquisas foram realizadas), tem-se que 6,3\% ocorreram na Ásia (2/32); 59,4\%, na América do Sul (19/32); 9,4\%, no Oriente Médio (3/32); 12,5\%, na Europa $(4 / 32) ; 3,1 \%$, na África $(1 / 32)$ e $9,4 \%$, na
América do Norte (3/32). As pesquisas relacionadas à exposição aos riscos biológicos concentraram-se mais na América do Sul e na Europa.

No que se refere às recomendações sobre biossegurança para os profissionais que atuam na atenção primária, os artigos apresentaram a preocupação em demonstrar os riscos aos quais estes profissionais estão expostos e poucos abordaram aspectos relacionados à segurança na execução das atividades. Quando essas são abordadas, fazem referência sempre à importância de treinamentos e capacitações constantes.

Os riscos ocupacionais biológicos estão diretamente ligados à falta de treinamento e de capacitação aos profissionais da saúde e, portanto, devem fazer parte da rotina de trabalho desses para permitir uma reflexão sobre a realidade vivenciada e auxiliar na mudança de comportamentos ${ }^{(24)}$.

\section{Conclusão}

Concluindo a presente revisão integrativa, em busca da melhor evidência disponível no mundo em relação à biossegurança na atenção primária à saúde e às recomendações para um trabalho seguro, entende-se que as produções científicas são escassas, apesar de nos últimos anos, ter havido aumento no número de publicações.

Os poucos estudos existentes apontam para a dificuldade de os profissionais da 
atenção primária aderir às precauções padrão, principalmente no que se refere à imunização. Não há controle de sorologia após a vacinação, o que os expõe a riscos no exercício de suas atividades laborais.

Outro dado que chama a atenção são os acidentes com objetos perfurocortantes, com maior exposição dos membros superiores, principalmente as mãos. Isso ocorre em razão de esses profissionais não adotarem as medidas de proteção adequadas na hora do descarte de agulhas. Existe, ainda, a não adesão ao uso de luvas, o que contribui para sua exposição aos agentes biológicos, considerando que, a maioria dos procedimentos por eles realizados, levam ao contato com algum tipo de fluido orgânico.

A prevalência dos acidentes com exposição aos agentes biológicos na atenção primária se assemelha às que ocorrem com os profissionais no ambiente hospitalar, explicitando a necessidade de um olhar mais aprofundado para adoção de medidas que possam proteger esses trabalhadores.

Evidenciou-se que a falta de treinamentos e/ou de capacitação desses profissionais também contribui para ampliar a possibilidade de exposição aos agentes biológicos durante as atividades, principalmente aquelas de contato direto com sangue ou fluidos corporais.
Considerando a importância que a atenção primária à saúde tem para a sociedade e a efetivação das políticas públicas de saúde no país, é fundamental que se estabeleçam estratégias que possam garantir um ambiente de trabalho isento de riscos às pessoas que atuam nessa área, com oferta de equipamentos de proteção e treinamentos/capacitações, minimizando assim os riscos ocupacionais inerentes à profissão.

Reafirma-se, então, a importância de serem estabelecidos programas de capacitação permanente aos profissionais da atenção primária para que possam adotar comportamentos de proteção individual e coletiva durante o trabalho.

Uma das limitações encontradas no desenvolvimento desse estudo foi à dificuldade de encontrar, na literatura mundial, artigos que abordassem a biossegurança e $\mathrm{o}$ risco ocupacional envolvendo todos os profissionais que atuam na atenção primária. Os existentes enfocam as categorias de forma isolada, o que dificulta comparar o nível de exposição entre essas categorias.

Isso reforça, ainda mais, a necessidade de se desenvolverem estudos que busquem fazer essa relação para que medidas de proteção possam ser planejadas e implantadas, de forma contextualizada, voltadas para as necessidades de cada 
profissão e seu nível de exposição, especificamente, na atenção primária.

\section{Referências}

1. Ministério da Saúde (BR). Conselho Nacional de Secretários de Saúde. Atenção primária e promoção da saúde. Brasília: CONASS; 2011.

2. Ministério da Saúde (BR). Secretaria de Atenção à Saúde. Departamento de Atenção Básica. Procedimentos. Brasília: Ministério da Saúde; 2011.

3. Monteiro ALC, Ruiz EAC, Paz RB. Recomendações e condutas após exposição ocupacional de profissionais de saúde. Bol Epidemiol CRT/AIDS CVE. 1999;17(1):3-23.

4. Association for Professionals in Infection Control and Epidemiology. APIC position paper: prevention of device mediated bloodborne infections to health care workers. Am J Infect Control. 1998 ;26(6):578-80.

5. Cardo DM, Culver DH, Ciesielski CA, Srivastava PU, Marcus R, Abitebou D, et al. A case-control study of HIV seroconversion in health care workers after percutaneous exposure. New Engl J Med. 1997;337(21):1485-90.

6. Bell DM. Occupational risk of human immunodeficiency virus infection in healthcare workers: an overview. Am J Med. 1997;102(Suppl 5B):9-15.

7. Henry K, Campbell S. Needlestick/sharps injuries and HIV exposure among health care workers: national estimates based on a survey of U.S. hospitals. Minn Med. 1995;78(11):41-4.

8. Canini SRMS, Gir E, Hayashida M, Machado AA. Acidentes perfurocortantes entre trabalhadores de enfermagem de um hospital universitário do interior paulista. Rev Latinoam. Enferm. 2002;10(2):172-8.

9. Joveleviths D, Schneider LO. Análise de acidentes de trabalho com materiais biológicos em hospital cirúrgico. Rev Anamt. 1996;9(1):4-5.

10. Santos NJS, Monteiro ALC, Ruiz EAC. The first case of AIDS due to occupational exposure in Brazil. Braz $\mathbf{J}$ Infec Dis. 2002;6(3):140-1.

11. Carvalho VG, Maciel DN, Hoefel MG, Yates ZB, Viana MC, Trindade DM. Perfil de aderência ao controle/seguimento após acidente com material biológico em um hospital universitário. Rev HCPA. 2002;22(supl):254.

12. Ministério do Trabalho (BR). Norma Regulamentadora $n^{\circ}$ 9: Programa de 
Prevenção dos Riscos Ambientais.

Brasília: Ministério do Trabalho; 1997.

13. Marinho J. Profissão perigo. CORENSP. 2004;(52): 7-11, 2004.

14. Cochrane AL. Effectiveness and efficiency: random reflections on health services. London: Nuffield Provincial Hospitals Trust; 1972.

15. Centers for Disease Control and Prevention. Immunization of health care workers: recommendations of the Advisory Committee on Immunizations Practice (ACIP) and the Hospital Infection Control Practices Advisory Committee (HICPAC). MMWR Recomm Rep. 1997:46(RR18):1-42.

16. Centers for Disease Control and Prevention. Updated U.S. Public Health Service Guidelines for the Management of Occupational Exposures to $\mathrm{HBV}, \mathrm{HCV}$, and $\mathrm{HIV}$ and Recommendations for Postexposure Prophylaxis. MMWR Recomm Rep. 2001;50(RR-11):1-42.

17. Tan L, Hawk JC 3rd, Sterling ML. Preventing needlestick injuries in health care settings. Arch Intern Med. 2001;161(7):929-36.

18. Alcântara CCS, Fonseca FGC, Albuquerque ABB, Ramos ANJ.
Riscos ocupacionais na atenção primária à saúde: susceptibilidade às doenças imunopreveníveis dos profissionais que atuam em uma unidade básica de saúde de Fortaleza, Ceará - 2003. Rev APS. 2005;8(2):143-50.

19. Whittemore R, Knafl K. Uma revisão integrativa: uma metodologia atualizada. $\quad$ J $\quad A d v \quad$ Nurs. 2005;52(5):546-53.

20. Stetler CB, Brunell M, Giuliano KK, Morsi D, Prince L, Newell-Stokes V. Evidence-based practice and the role of nursing ledership. J Nurs Adm. 1998;28(7-8):45-53.

21. Sarquis LMM, Felli, VEA. Acidentes de trabalho com instrumentos perfurocortantes entre os trabalhadores de enfermagem. Rev Esc Enferm USP. 2002;36(3):222-30.

22. Caixeta RB, Barbosa-Branco, A. Acidente de trabalho, com material biológico, em profissionais de saúde de hospitais públicos do Distrito Federal, Brasil, 2002/2003. Cad Saúde Pública. 2005;21(3):737-46.

23. Souza ELV, Nascimento JC, Caetano JA, Enfermeira RCVR. Uso dos equipamentos de proteção individual em unidade de terapia intensiva. Rev Enf Ref [Internet]. 2011 [citado 2 jan 
2014];3(4):125-33. Disponível em:

http://www.scielo.gpeari.mctes.pt/sciel

o.php?pid=S0874-

02832011000200013\&script=sci_artte

$\mathrm{xt}$
24. Miranda EJP, Stancato K. Riscos à saúde de equipe de enfermagem em unidade de terapia intensiva: proposta de abordagem integral da saúde. Rev Bras Ter Intensiva. 2008;20(1):68-76. 